\title{
Parameters Study on Precise Straightening Process in Roll Straightener
}

\author{
Qiang Chen ${ }^{1, a}$, Shan $\mathrm{Li}^{1, \mathrm{~b}}$, Ying $\mathrm{LuO}^{2, \mathrm{c}}$ and Wenlong $\mathrm{Lu}^{1, \mathrm{~d}}$ \\ ${ }^{1}$ Mechanical and Electrical Engineering College, Kunming University of Science and \\ Technology,kunming,China \\ 2 Shenji Group Kunming Machine Tool Co., Ltd.,kunming,China \\ a544280704@qq.com, b24814911@qq.com, ${ }^{\text {'5 } 568555591 @ q q . c o m, ~ I w l j i n g @ q q . c o m ~}$
}

Keywords: Roller Leveler; Copper Starting Sheet; Precise Straightening; Simulation

Abstract. Using electrolysis method can produce high quality of refined copper, copper cathode plate applied to the electrochemical machining. In order to make the copper cathode in electrolysis with high flatness to ensure distance between cathode and anode plate, so that purity cathode copper higher, is committed to meet the conditions of the electrolytic process of copper cathode, so change the straightening plates became an urgent task of the enterprise. ANSYS/LS-DYNA by computer simulation and computer optimization. Straighteners provide reference for the design and development of the enterprise, it has practical value and significance.

\section{Introduction}

At present, China's smelting mostly refined copper of high purity by electrolysis method, because the electrolysis can gain levels above $99.9 \%$ of refined copper, and can only fire refining copper content was maintained at $99 \%$ to $99.8 \%$. If the verticality of the cathode and anode plates is not high, a direct impact is the pole pitch. Pole pitch change makes the plates through a current density inhomogeneity, and thus make thickness of the copper precipitation inhomogeneity, so that current consumption levels are reduced [1,2]. But the smaller pole spacing also makes it easier to have a short circuit phenomenon is "burning board", appear a lot of crystallization in the short, electricity use efficiency decreased, and the cathode plate cannot be produced to meet production requirements.
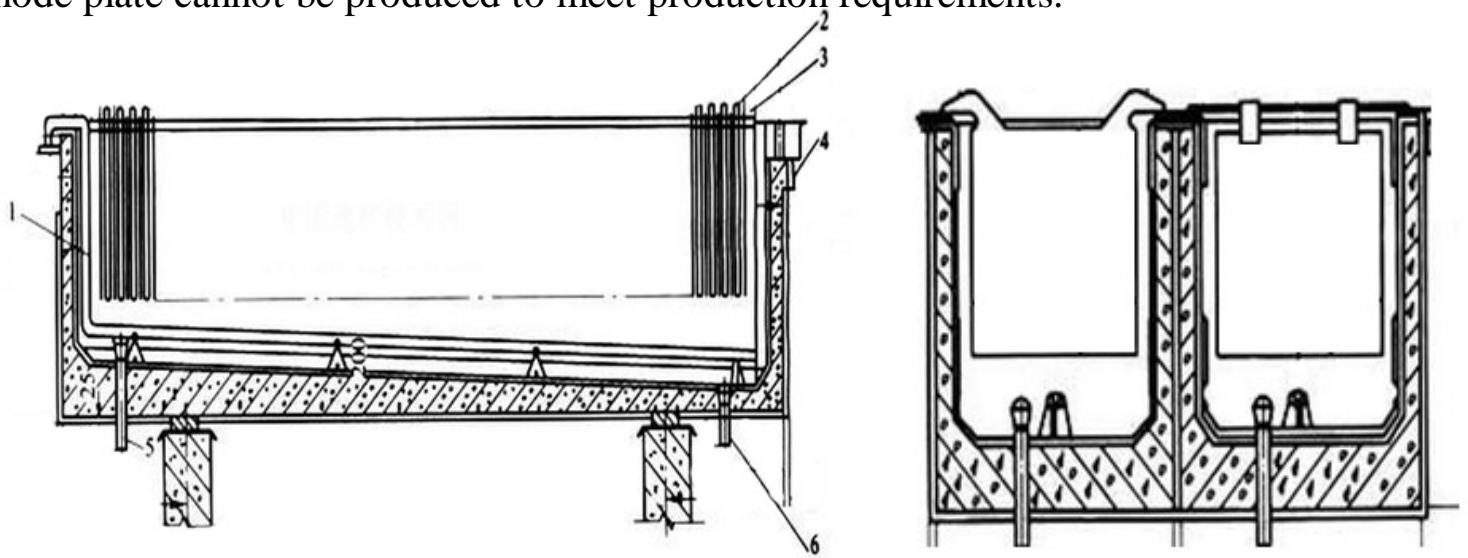

Figure 1 the copper electrolytic cell schematic

1-liquid inlet pipe; 2 - the anode plate; 3-the negative plate; 4 - liquid outlet pipe; 5 - liquid discharge pipe; 6- extrude anode mud outlet pipe

\section{Research contents}

This paper studies the starting sheet for reinforced rectangular plate of copper, and its size is as follows: $820 \pm 10 \mathrm{~mm}$ long, $720 \pm 10 \mathrm{~mm}$ wide and $0.8 \mathrm{~mm}$ thick. By measuring the rough after straightening the starting sheet of copper, it could obtain that the volatility of copper starting sheet was $6.8 \mathrm{~mm}$, and the wavelength of it was $80 \mathrm{~mm}$. According to modern straightener of changing requirements and the actual production conditions, the actual production situation, to strengthen the state copper starting sheet as, using the mathematical model to the precision straightening Maple 
software program. Precision mathematical model of straightening with the Maple software is programmed by Newton-Raphson method to calculate the number of fine straightening rollers in the appliance. Now we choose four different sets of roller spacing parameters, three sets of changing linear velocity. Match the pitch and changing to straight line speed, divided into 12 groups of computer modeling and simulation. Observation of copper starting sheet straightening effect and select the most reasonable parameter group in order to provide references for enterprises, and the roller diameters corresponded to the selected roller pitches were $p=20 \mathrm{~mm}, p=30 \mathrm{~mm}, p=40 \mathrm{~mm}, p=50 \mathrm{~mm}$.

Because the linear velocity of the straightening should not be too large, so the linear velocity of the straightening parameters selection were $\mathrm{v}=200 \mathrm{~mm} / \mathrm{s}, \mathrm{v}=250 \mathrm{~mm} / \mathrm{s}, \mathrm{v}=300 \mathrm{~mm} / \mathrm{s}$.

In this paper, with a row of roller fixed, another row roller adjustable arrangement as the research object. The bottom roller is fixed, on top of roller is adjustable. The intermeshes in different roller pitches of the top rollers in precise straightening device were calculated by using the bending curvature ratio and the elastic limit deflection of copper starting sheet, the values of the intermesh were shown in Table 1, Table 2, Table 3 and Table 4.

Table $1 \mathrm{p}=20 \mathrm{~mm}$, the intermesh of the top rollers in precise straightening device

\begin{tabular}{|c|c|c|c|c|c|}
\hline Roller No. $i$ & 2 & 4 & 6 & 8 & 10 \\
\hline Intermesh $\delta_{i}$ & 0.050 & 0.037 & 0.032 & 0.030 & 0.030 \\
\hline \multicolumn{6}{|c|}{ Table $2 \mathrm{p}=30 \mathrm{~mm}$, the intermesh of the top rollers in precise straightening device } \\
\hline Roller No. $i$ & 2 & 4 & 6 & 8 & 10 \\
\hline Intermesh $\delta_{i}$ & 0.114 & 0.086 & 0.075 & 0.070 & 0.068 \\
\hline \multicolumn{6}{|c|}{ Table $3 \mathrm{p}=40 \mathrm{~mm}$, the intermesh of the top rollers in precise straightening device } \\
\hline Roller No. $i$ & 2 & 4 & 6 & 8 & 10 \\
\hline Intermesh $\delta_{i}$ & 0.200 & 0.150 & 0.132 & 0.124 & 0.118 \\
\hline \multicolumn{6}{|c|}{ Table $4 \mathrm{p}=50 \mathrm{~mm}$, the intermesh of the top rollers in precise straightening device } \\
\hline Roller No. $i$ & 2 & 4 & 6 & 8 & 10 \\
\hline Intermesh $\delta_{i}$ & 0.314 & 0.237 & 0.207 & 0.193 & 0.186 \\
\hline
\end{tabular}

\section{The simulation analysis of precise straightening process}

For roller spacing is $20 \mathrm{~mm}, 30 \mathrm{~mm}, 40 \mathrm{~mm}, 50 \mathrm{~mm}$ respectively match three different linear velocity correction, which is $200 \mathrm{~mm} / \mathrm{s}, 250 \mathrm{~mm} / \mathrm{s}, 300 \mathrm{~mm} / \mathrm{s}$. Three different linear velocities parameters and four different roller pitches parameters were separately matched and combined into 12 groups of matching parameters. The twelve sets of parameters for simulation in ANSYS/LS-DYNA[3,4]. In the process of modeling, from refined correcting roller and copper starting sheet part of the analysis, was observed when straightening the straight-line speed were taken $\mathrm{v}=200 \mathrm{~mm} / \mathrm{s}, \mathrm{V}$ equals $250 \mathrm{~mm} / \mathrm{s}$, $\mathrm{v}=300 \mathrm{~mm} / \mathrm{s}$ copper starting sheet on the same node straightening straight change situation.

Firstly, the model of the copper starting sheet and the precise straightening roll is established in ANSYS/LS-DYNA, and the load of the twelve sets of models are calculated respectively. The file plot into Isprepost.exe after operation, we can observe roller straightening machine the copper starting sheet process [5]. Figure 2 shows the copper starting sheet initial, bite, precise straightening and end of the four stages.

After copper starting sheet precise straightening is complete, copper starting sheet were observed at the level of speed $200 \mathrm{~mm} / \mathrm{s}, 250 \mathrm{~mm} / \mathrm{s}, 300 \mathrm{~mm} / \mathrm{s}$ when the deformation and residual stress of the situation in the Y direction. By maximum deformation Ymax, minimum deformation Ymin, copper 
starting sheet of the original amplitude $\mathrm{Rv}=6.8 \mathrm{~mm}$. The copper starting sheet of residual or too large deformation for out ratio with the initial amplitude of of $\mathrm{R} \mathrm{V}$, cartculate the final copper starting

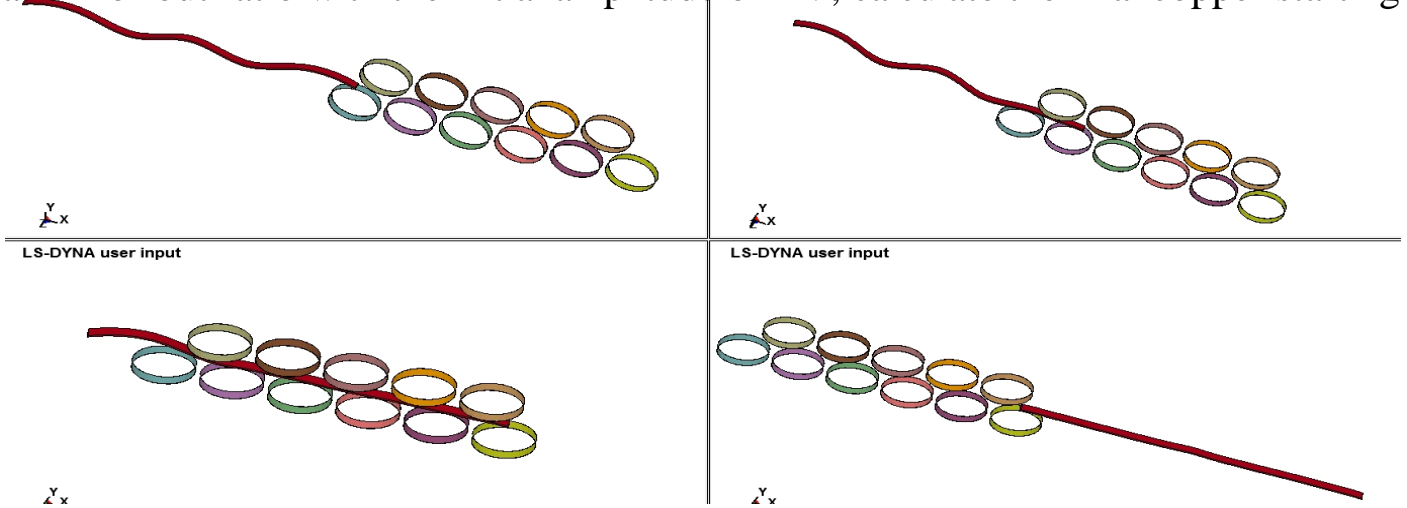

Fig.2each stage of precise straightening copper starting sheet

sheet straightening residual percentage. In order to find the most suitable matching parameter, the percentage of the residual rate of straightening is measured. When $\mathrm{p}=40 \mathrm{~mm}$ and the precise straightening $\mathrm{v}=250 \mathrm{~mm} / \mathrm{s}$, the deformation condition of copper starting sheet in Y direction and the maximum and minimum deformation values were shown in Figure 3 and the residual stress changes of copper starting sheet and the maximum and minimum values of residual stress were shown in Figure 4 .

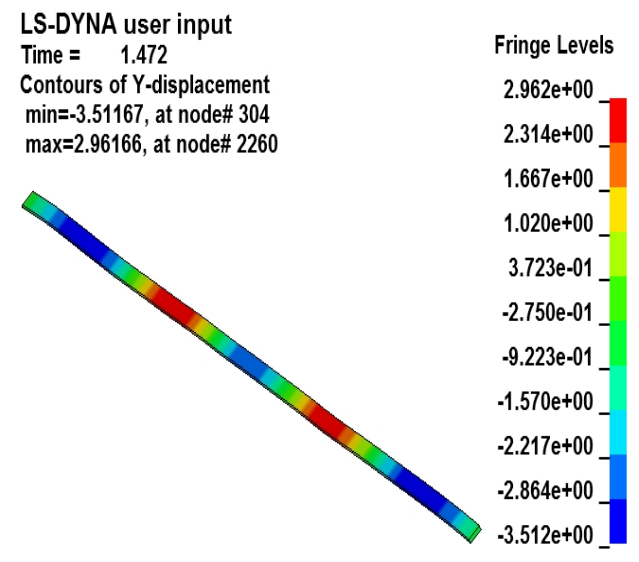

Fig. $3 \mathrm{p}=40 \mathrm{~mm}, \mathrm{v}=250 \mathrm{~mm} / \mathrm{s}$, Y-deformation of copper starting sheet

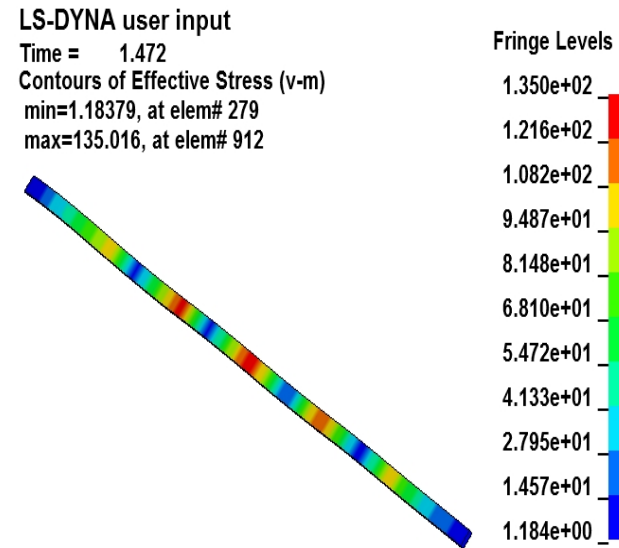

Fig. $4 \mathrm{p}=40 \mathrm{~mm}, \mathrm{v}=250 \mathrm{~mm} / \mathrm{s}$, residual stress of copper starting sheet

Observed of figure 3, figure 4 , when the roller pitch is $40 \mathrm{~mm}$, the linear velocity is $250 \mathrm{~mm} / \mathrm{s}$, the percentage of residual curvature copper starting sheet is $4.8 \%$, the maximum residual stress is 135.016 Pa.

The percentage of residual rate of copper starting sheet is increased with the increase of the precise straightening. This is caused by the unstable precise straightening, the residual stress increases with the increase of the precision straightening velocity. The smaller the residual rate percentage value was, the better the precise straightening was, and the higher the flatness of copper starting sheet was.

\section{Statistical analysis}

The Y deformation and residual stress data obtained from the simulation are calculated. A list of each parameter corresponding to the copper startingsheet final residue percentage and maximum residual stress. Through the analysis of these two kinds of statistics, we find out the best matching parameters of the precision and efficiency, the best matching parameters and the residual stress parameters.

Through the above analysis, we can know that when $p=20 \mathrm{~mm}, \mathrm{v}=200 \mathrm{~mm} / \mathrm{s}$, the precise straightening has the best percentage of residual rate, but the maximum residual stress is larger; When $\mathrm{p}=20 \mathrm{~mm}, \mathrm{v}=300 \mathrm{~mm} / \mathrm{s}$, the precise straightening has the best precision efficiency. When $\mathrm{p}=50 \mathrm{~mm}$ 
, $v=200 \mathrm{~mm} / \mathrm{s}$, the precise straightening has the best residual stress, but the residual rate of fine correction is quite large.

Table 5 the comparison table of matching program

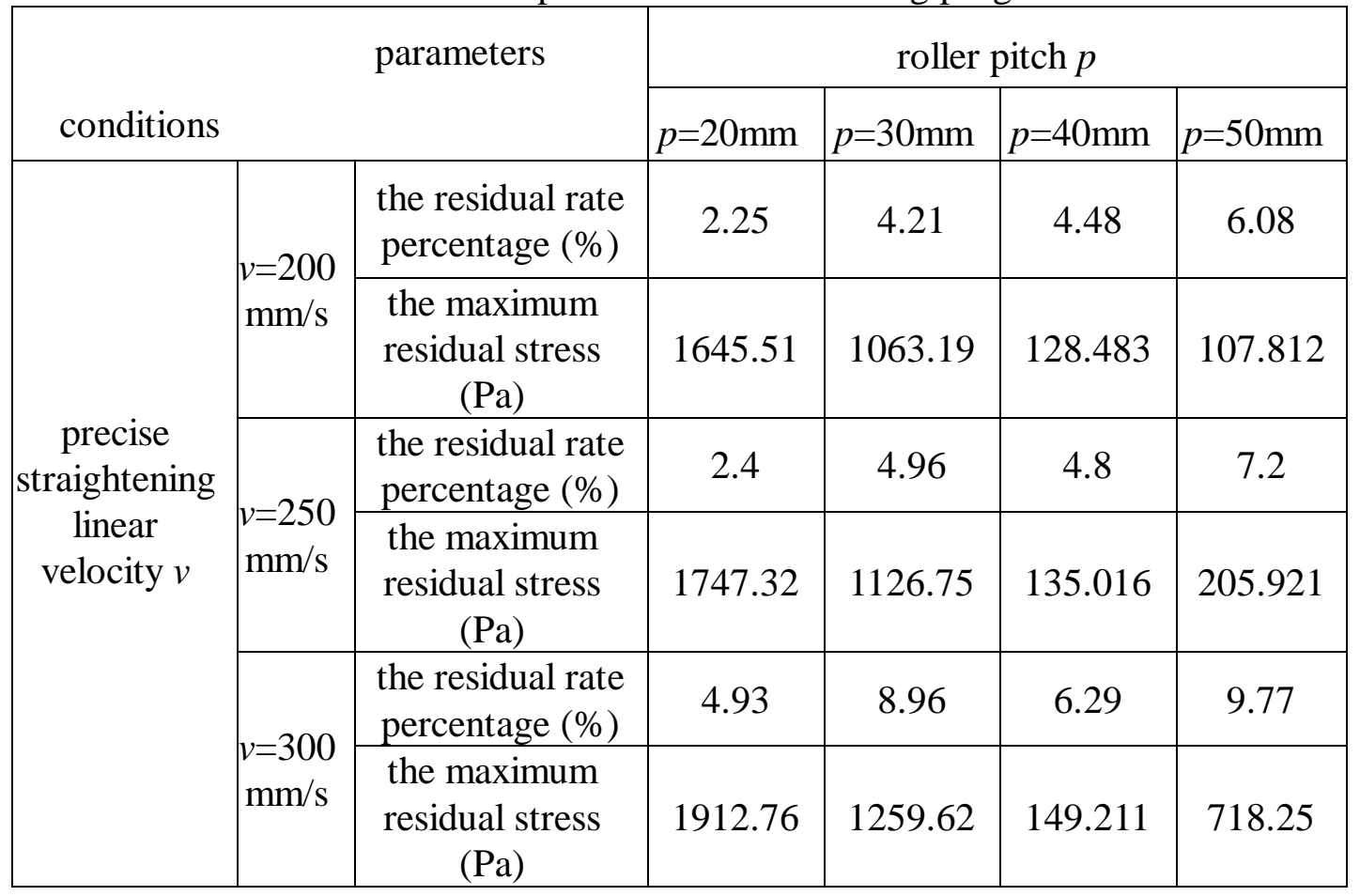

\section{Conclusion}

The parameters of the four sets of roller pitch and the three groups precise straightening velocity were matched with each other. Twelve groups of parameters were analyzed in ANSYS/LS-DYNA. Through the analysis of the twelve groups of parameters in the precision of the time, the percentage of residual rate, after finishing the copper, the maximum residual stress of the copper starting sheet. Find the most reasonable set of parameters, that is $\mathrm{p}=40 \mathrm{~mm}, \mathrm{v}=250 \mathrm{~mm} / \mathrm{s}$. If the reasonable parameter is extended to a certain range, then roller pitch from 30 to $40 \mathrm{~mm}$, precise straightening linear velocity of $200-250 \mathrm{~mm} / \mathrm{s}$, in this interval value to copper starting sheet have reached a good level of the Precise straightening effect and residual stress.

\section{Acknowledgements}

This work was financially supported by the "high-end CNC machine tools and basic manufacturing equipment" major science and technology issues" precision vertical and horizontal machining center technology innovation platform" (Item No.2012ZX04012-031, January 2012 to December 2015)

\section{References}

[1] Carl Vananda. Challenges of the Second Industrial Revolution. The New York Times Magazine, Vol.5(1961),P51-53

[2] Yongxiang $\mathrm{Qu}$. To build a strong power of copper industry. China nonferrous metals, vol.1(2012),P42-43

[3] Yuchun Li,Dangyong Shi: ANSYS11.0/LS-DYNA Basic theory and engineering practice(2008)

[4] J.O. HALLQUIST. LS-DYNA Theoretical Manual. Livermore Software Techhnology Corporation(1998) 
[5] Polyakov, B N. Parameters of Roller Straightening Machines. Steel in Translation, Vol.9(2006),P53-56 